\title{
The impact of telehealth on patient attendance and revenue within an Aboriginal Community Controlled Health Organisation during COVID-19
}

Danielle Couch, Zakary Doherty, Laura Panozzo, Thileepan Naren, Jaydene Burzacott, Bernadette Ward, Rebecca Kippen, Dallas Widdicombe

\section{Background and objective Aboriginal Community Controlled Health Organisations (ACCHOs) provide culturally appropriate medical services to Aboriginal and/or Torres Strait Islander people. The aim of this study was to examine the impact of telehealth on patient attendance and revenue within an ACCHO during COVID-19.}

\section{Methods}

This is a time-series study of general practitioner attendances at a regional Victorian ACCHO in two periods: March-June 2019 (pre-COVID-19) and March-June 2020 (during COVID-19).

\section{Results}

After adjusting for the number of available appointments, there was a $27 \%$ increased rate of attendances per appointment slot during the COVID-19 period when compared with the preCOVID-19 period, and a 59\% increase in Medicare Benefits Schedule items claimed during the COVID-19 period, compared with the pre-COVID-19 period.

\section{Discussion}

The findings indicate that the provision of services via telehealth increased the number of people able to access the medical clinic, and that this had a positive financial impact for the organisation.
ABORIGINAL COMMUNITY CONTROLLED HEALTH ORGANISATIONS (ACCHOs) play a pivotal part in the provision of culturally appropriate, locally based and accessible health and medical services to Aboriginal and Torres Strait Islander Australians. ${ }^{1}$ Chronic disease management and prevention activities delivered in ACCHOs appear to be equal to, if not better than, care delivered by general practices. ${ }^{2}$ Telehealth can be defined as the 'use of telecommunication $[\mathrm{s}]$... for ... providing telemedicine, medical education and health education over a distance'. ${ }^{3}$ It has been long hoped that telehealth will become effectively integrated to allow the provision of timely, accessible and cost-effective health services, particularly to people living in rural areas. A 2017 systematic review of telehealth with Aboriginal and Torres Strait Islander communities highlighted benefits, such as social and emotional wellbeing, the advantages of receiving care in community, family attendance during care, options for palliative patients to die on Country, increased patient empowerment and improvements in personal wellbeing. ${ }^{4}$ There is growing evidence for the provision of telehealth to Aboriginal and Torres Strait Islander people, but most research has been focused on remote communities, and during times of 'business as usual', not during a pandemic, such as the COVID-19 pandemic.
In mid-March 2020, as a response to COVID-19 the Australian Government introduced new Medicare Benefits Schedule (MBS)-subsidised telehealth services and provided extra incentives to general practitioners (GPs) and other health practitioners. There were additional MBS item numbers for telehealth services with medical specialists and nurse practitioners, access for mental health treatments, chronic disease management and Aboriginal and Torres Strait Islander Health assessments. ${ }^{5}$ National social distancing restrictions were eased from mid-May 2020 but re-introduced in the state of Victoria from late June 2020 because of a local spike in cases. MBS-subsidised telehealth services are still in place as of October 2021. The COVID-19 pandemic has forced many general practices to rapidly transition to using telehealth models of care as a means of caring for patients and minimising the risks of transmitting COVID-19. Responding to the pandemic and these changes, ACCHOs in many locations increased the number and type of services they were delivering via telehealth, particularly for general practice-related services.

The swift uptake of telehealth has changed the paradigm of how general practice care is delivered, but to date, there has been limited research into how ACCHOs have used telehealth technology 
and any impacts on patient attendance during COVID-19. The rapidly changing legislative and regulatory environment surrounding telehealth warrants further investigation of this modality of delivering healthcare in ACCHOs.

The aim of this study was to examine the impact of telehealth on patient attendance and revenue within a regional ACCHO during COVID-19.

\section{Methods}

\section{Study concept and design}

In line with self-determination and data sovereignty, ${ }^{6,7}$ this project was developed and led by a regional ACCHO, Bendigo \& District Aboriginal Co-operative (BDAC). Founded in 2001, BDAC is based in Bendigo, Victoria, and is the only ACCHO providing services across Dja Dja Wurrung Country. Services are embedded in a comprehensive and integrated model of primary healthcare, ${ }^{8}$ informed by a social and cultural determinants framework, ${ }^{9}$ and specifically designed to meet the needs of Aboriginal and/or Torres Strait Islander people in the area.

A time-series study was used to examine all general practice clinic attendances at BDAC during two distinct periods: 1 March - 30 June 2019 (pre-COVID-19) and 1 March - 30 June 2020 (during COVID-19).

\section{Study setting}

Approximately 1450 patients access BDAC's medical clinic annually. In 2019, BDAC had two full-time equivalent GPs and one sessional GP. In February 2020, this expanded to include an additional part-time general practice registrar. The introduction of telehealth via telephone and video calls was among the many changes BDAC made in response to the COVID-19 pandemic, including stricter social distancing protocols and allowing community members to be on site at certain times. Most telehealth appointments were by telephone, consistent with telehealth uptake across general practice more generally. ${ }^{10}$ There were minimal costs associated with the change as the HealthDirect telehealth platform was free, ${ }^{11}$ and no extra staff were hired to support telehealth specifically. There were some small additional costs in terms of hardware upgrades (eg dual monitors, camera and speakers), but these were included in the organisation's wider facilities and assets expenses, as the whole organisation was moving to supporting remote access and work-from-home options in response to the pandemic.

\section{Data source}

Attendance data were sourced from an internal BDAC database. For each weekday of the study period, a summary of the claimed MBS item numbers was recorded. Weekends and public holidays were not included as the clinic is not open on those days. Data on the number of appointments (a 20-minute appointment slot) available for bookings on each day were also extracted.

\section{Variables}

MBS items were first examined by day and GP, and then aggregated into daily summary data. Items for 'standard' attendances were the focus of this work. Standard attendances were defined as face-to-face or telehealth appointments that were general in nature. Each MBS item code was assumed to correspond with a single unique attendance. Specific MBS items for mental health consults, procedures, facility visits or GP management plans were not included. Further information regarding categorisation of MBS items is available in Appendix 1 (available online only). Non-attended appointments were excluded as there was no MBS item claimed for these.

\section{Outcomes}

The primary outcome was the attendance rate per available appointment during each time period. The secondary outcome was the revenue generated per appointment in each time period.

\section{Statistical analysis}

To control for staffing changes and a difference in the number of days the clinic was open between the 2019 and 2020 data periods, the number of attendances (as defined by an MBS code) in each category were divided by the number of appointment slots available in the relevant week. This resulted in a rate of attendance per appointment slot being calculated for each month. Revenue amounts were calculated by using the MBS website to identify the rebate value for each code in Australian dollars. To facilitate comparison, 2020 values were used. ${ }^{12}$

Negative binomial regression was used to assess any significant change in the number of attendances per appointment slot per month. This regression method determines what the average change in the rate (attendances per appointment slot) was per unit of time (month in this study), and if such a change was significant. A $P$ value of $<0.05$ was deemed statistically significant. All analyses were conducted using $\mathrm{R}$ version 4.0.2. ${ }^{13}$

The project was approved by the Monash University Human Research Ethics Committee (ID: 26526). The database did not include any identifying patient information.

\section{Results}

During the pre-COVID-19 (2019) and COVID-19 (2020) periods, the clinic was open for 86 and 87 days, respectively. As a result of lower staff numbers, there were fewer available appointment slots offered during the pre-COVID-19 period when compared with the COVID-19 period (3348, compared with 4186).

A total of 1750 MBS standard items were claimed for attendances during the pre-COVID-19 period, while 2783 were claimed during the COVID-19 period, representing a $59 \%$ increase (Figure 1).

The rate of standard attendances per appointment slot increased from 0.52 in the pre-COVID-19 period to 0.66 in the COVID-19 period - an overall $27 \%$ increase. The increase per month was 5.5\% (95\% confidence interval: 4, 7; $P<0.001)$. During the COVID-19 period, $44 \%$ of standard attendances were conducted via telehealth, compared with no standard attendances conducted via telehealth in the pre-COVID-19 period.

Clinic revenue from MBS billings also increased between the periods. Revenue increased by $17 \%$, with $\$ 26.4$ generated 
per appointment slot in the pre-COVID-19 period, compared with $\$ 30.8$ generated during the COVID-19 period (Figure 2).

\section{Discussion}

Regular access to primary care is imperative for improving health outcomes and supporting health equity, ${ }^{14}$ and ACCHOs play a key part in the provision of this care to Aboriginal and/or Torres Strait Islander people in Australia. ${ }^{2}$ While the use of telehealth in ACCHOs is not new, COVID-19 has forced the large-scale adoption of this technology. This study examined telehealth attendance and revenue in a regional Victorian ACCHO during COVID-19.

Prior to COVID-19, no standard appointments at BDAC were conducted via telehealth, whereas during the COVID-19 period, $44 \%$ of standard attendances were conducted via telehealth. During the COVID-19 period, there was a $27 \%$ increase in the rate of attendances per appointment slot, which can be interpreted as each available booking slot was occupied with an attendance $27 \%$ more often in the COVID-19 period. This suggests that the provision of services via telehealth increased the number of people able to access the clinic and medical services.

Given the well-known health 'gap' experienced by Aboriginal and Torres Strait Islander people, alternative forms of providing access to primary care, such as telehealth, may provide an important opportunity for addressing the gap and for ensuring primary care is available to communities who may be harder to reach. ${ }^{15}$ This is consistent with other evidence highlighting the beneficial impacts of telehealth on the provision of health services and different models of care. ${ }^{16,17}$

ACCHOs are increasingly under pressure to be financially 'sustainable' and are encouraged to diversify income streams while still providing quality care to patients. The results of the present study found an increase in revenue of $17 \%$ per appointment slot during the COVID-19 period, indicating that the provision of services via telehealth had a positive financial impact for the organisation. There were some minimal one-off costs to set up telehealth delivery, which the organisation absorbed as part of its wider asset upgrades required in response to COVID-19.

An ongoing opportunity to deliver some services via telehealth may offer income diversification opportunities for ACCHOs. It is common for ACCHOs to support patients with greater and increased complexity of care needs, and ACCHO GPs also manage a larger number of problems per consultation, ${ }^{18}$ with this work not appropriately or sufficiently rewarded within the current time-based MBS structure. It is well known that mainstream programs, such as the MBS, fail to adequately cater for Aboriginal and Torres Strait Islander people, and quantifying the economic value

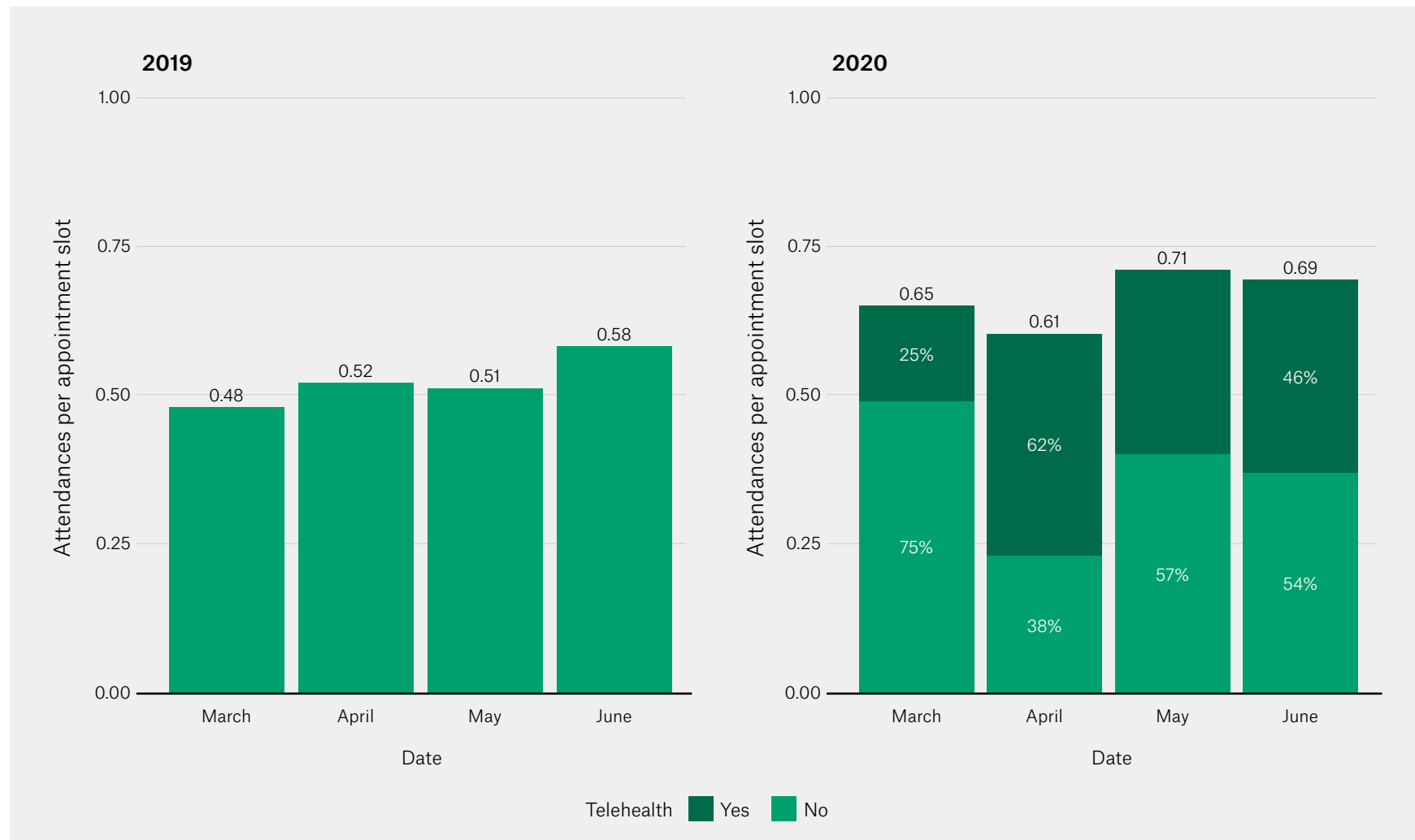

Figure 1. Claims per appointment slot by month, with stratification by whether appointment was done via telehealth 
of ACCHOs is difficult. ${ }^{19}$ The 20 -minute appointment slots that BDAC provides for patients are not a financially viable model of practising general practice under the current MBS payment structure but are required and appropriate because of the aforementioned factors. The continuation of the telehealth MBS items introduced in March 2020 beyond COVID-19 would provide an opportunity to continue to fund the care ACCHOs provide in supporting patients with greater needs, which is often not replicated in mainstream practices.

A limitation of this study is the small sample size and the focus on only standard appointments. The small sample size restricted the ability to investigate changes in other appointment types (the excluded appointment types were facility visits, GP management plans, mental health and procedures). Additionally, correlation over time does not necessarily imply causation, although the statistical association is indicative that the introduction of new telehealth services resulted in increased attendances and income.

Future research should investigate clinical outcomes associated with increased patient attendance at clinics, and the perceptions and experiences of Aboriginal and Torres Strait Islander patients and ACCHO GPs in relation to the benefits and challenges of medical care provision via telehealth.

\section{Conclusion}

Rapid implementation of telehealth within a regional Victorian ACCHO was associated with a significant increase in standard attendances at the medical clinic and increased practice income. The ongoing availability of telehealth consultations continuing in an unrestricted manner for ACCHOs beyond COVID-19 is an important adjunct to holistic general practice and comprehensive primary care that ACCHOs provide, and will serve to aid with continuity of care and improvement of access to primary healthcare services.

\section{Authors}

Danielle Couch PhD, Strategic Projects/Policy Adviser, Bendigo \& District Aboriginal Co-operative, Bendigo, Vic; Adjunct Research Fellow, School of Rural Health, Monash University, Bendigo, Vic

Zakary Doherty BMedSc (Hons), MD, Research Fellow, School of Rural Health, Monash University, Bendigo, Vic

Laura Panozzo BMedSc (Hons), MD, Research Fellow, School of Rural Health, Monash University, Bendigo, Vic
Thileepan Naren MBBS, MHM, MPH, FACRRM, FRACGP, GAICD, General Practitioner, Bendigo \& District Aboriginal Co-operative, Bendigo, Vic

Jaydene Burzacott, Aboriginal Health Practitioner/ Clinical Team Leader, Bendigo \& District Aboriginal Co-operative, Bendigo, Vic

Bernadette Ward PhD, Associate Professor, School of Rural Health, Monash University, Bendigo, Vic

Rebecca Kippen PhD, Associate Professor of Demography, School of Rural Health, Monash University, Bendigo, Vic

Dallas Widdicombe BSW, Executive Director, Bendigo \& District Aboriginal Co-operative, Bendigo, Vic

Competing interests: DC is an employee of Bendigo \& District Aboriginal Co-operative, the study site. They had input into study design and development and manuscript preparation, but to minimise perceived conflict or influence, did not analyse the data. DC is also employed as a casual researcher by General Practice Supervisors Australia. RK reports grants from General Practice Supervisors Australia, the Avant Foundation, The Royal Australian College of General Practitioners and the Medical Research Future Fund, all outside the submitted work.

Funding: None.

Provenance and peer review: Not commissioned, externally peer reviewed.

Correspondence to:

danielle.couch@bdac.com.au

\section{Acknowledgements}

The authors acknowledge the ongoing sovereignty of Aboriginal and Torres Strait Islander Peoples across our lands, and pay their respect to Elders, past and present, and emerging. The authors would like to thank their patients at Bendigo \& District Aboriginal Co-operative who continued to seek care from them during COVID-19 and readily took to telehealth.

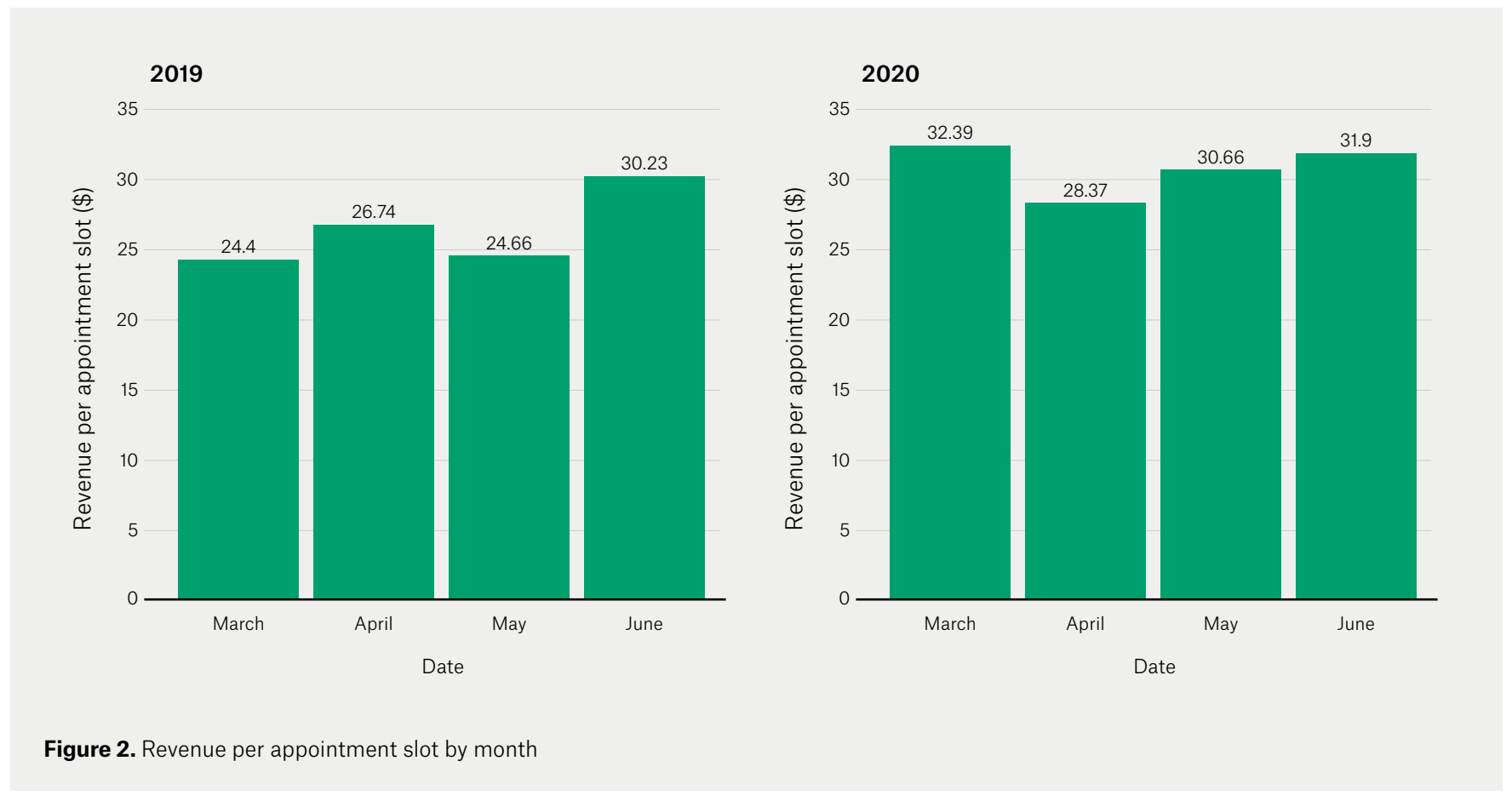




\section{References}

1. Khoury P. Beyond the biomedical paradigm: The formation and development of Indigenous community-controlled health organizations in Australia. Int J Health Serv 2015;45(3):471-94. doi: 10.1177/0020731415584557.

2. Panaretto KS, Wenitong M, Button S, Ring IT. Aboriginal community controlled health services: Leading the way in primary care. Med J Aust 2014;200(11):649-52. doi: 10.5694/mja13.00005.

3. Department of Health. Telehealth. Canberra, ACT: $\mathrm{DoH}, 2015$. Available at www1.health.gov.au/ internet/main/publishing.nsf/Content/e-healthtelehealth\#: :text=The\%2OInternational\%20 Organisation\%20for\%20Standardisation,as\%20 the\%20'use\%20of\%20advanced [Accessed 2 September 2021].

4. Caffery LJ, Bradford NK, Wickramasinghe SI, Hayman N, Smith AC. Outcomes of using telehealth for the provision of healthcare to Aboriginal and Torres Strait Islander people: A systematic review. Aust N Z J Public Health 2017:41(1):48-53. doi: 10.1111/1753-6405.12600.

5. Australian Government Department of Health. COVID-19: Whole of population telehealth for patients, general practice, primary care and other medical services [Media release]. 29 March 2020. Available at www.health.gov.au/ministers/ the-hon-greg-hunt-mp/media/covid-19-wholeof-population-telehealth-for-patients-generalpractice-primary-care-and-other-medical-services [Accessed 27 July 2020].
6. Kukutai T, Taylor J. Indigenous data sovereignty: Toward an agenda. Canberra, ACT: ANU Press, 2016

7. National Health and Medical Research Council. Keeping research on track II: A companion document to Ethical conduct in research with Aboriginal and Torres Strait Islander Peoples and communities: Guidelines for researchers and stakeholders. Canberra, ACT: NHMRC, 2018.

8. Aboriginal Health \& Medical Research Council of New South Wales. Aboriginal communities improving Aboriginal health: An evidence review of the contribution of Aboriginal community controlled health services to improving Aboriginal health. Sydney, NSW: AH\&MRC, 2015.

9. Australian Institute of Health and Welfare. 6.6 Social determinants and Indigenous health In: Australia's health 2018. Cat. no. AUS 221. Canberra, ACT: AlHW, 2018.

10. Snoswell CL, Caffery LJ, Haydon HM, Thomas EE, Smith AC. Telehealth uptake in general practice as a result of the coronavirus (COVID-19) pandemic. Aust Health Rev 2020;44(5):737-40. doi: 10.1071/ $\mathrm{AH} 20183$.

11. Healthdirect Australia. About Healthdirect video call. Haymarket, NSW: Healthdirect Australia, 2021. Available at about.healthdirect.gov.au/videocall [Accessed 9 August 2021].

12. MBS Online. MBS Online: Medicare Benefits Schedule. Canberra, ACT: Australian Government Department of Health, 2020. Available at www. mbsonline.gov.au/internet/mbsonline/publishing. nsf/Content/Home [Accessed 1 June 2021].
13. RStudio. R statistical computing software, version 4.0.2. Boston, US: The R Foundation, 2021.

14. Shi $L$. The impact of primary care: A focused review. Scientifica 2012;2012:432892. doi: 10.6064/2012/432892.

15. Australian Medical Association. AMA calls for introduction of permanent telehealth system [Media release]. 29 Apr 2021. Available at www. ama.com.au/gpnn/issue-21-number-16/articles/ ama-calls-introduction-permanent-telehealthsystem [Accessed 1 June 2021].

16. Monaghesh $E$, Hajizadeh A. The role of telehealth during COVID-19 outbreak: A systematic review based on current evidence. BMC Public Health 2020;20(1):1193. doi: 10.1186/s12889-020-09301-4.

17. Bradford NK, Caffery LJ, Smith AC. Telehealth services in rural and remote Australia: A systematic review of models of care and factors influencing success and sustainability. Rural Remote Health 2016;16(4):3808.

18. Larkins SL, Geia LK, Panaretto KS. Consultations in general practice and at an Aboriginal community controlled health service: Do they differ? Rural Remote Health 2006;6(3):560.

19. Alford K. Economic value of Aboriginal Community Controlled Health Organisations. Canberra, ACT: National Aboriginal Community Controlled Health Organisation, 2014.

correspondence ajgp@racgp.org.au 